Article

\title{
Integrative Analysis of Terpenoid Profiles and Hormones from Fruits of Red-Flesh Citrus Mutants and Their Wild Types
}

\author{
Cuihua Liu ${ }^{1,2}$, Min $\mathrm{He}^{2}$, Zhuang Wang ${ }^{2}$ and Juan $\mathrm{Xu}{ }^{2, *}$ \\ 1 College of Horticulture, Northwest A\&F University, Yangling 712100, China; liuch@nwafu.edu.cn \\ 2 Key Laboratory of Horticultural Plant Biology (Ministry of Education), College of Horticulture and Forestry, \\ Huazhong Agricultural University, Wuhan 430070, China; heminsweet@163.com (M.H.); \\ guhun_145@163.com (Z.W.) \\ * Correspondence: xujuan@mail.hzau.edu.cn; Tel.: +86-278-728-6965
}

Received: 16 August 2019; Accepted: 21 September 2019; Published: 23 September 2019

\begin{abstract}
In citrus color mutants, the levels of carotenoid constituents and other secondary metabolites are different in their corresponding wild types. Terpenoids are closely related to coloration, bitterness, and flavor. In this study, terpenoid profiles and hormones in citrus fruits of two red-flesh mutants-Red Anliu orange and Red-flesh Guanxi pummelo-and their corresponding wild types were investigated using GC/MS, HPLC, and LC-MS/MS. Results showed that Red Anliu orange (high in carotenoids) and Anliu orange (low in carotenoids) accumulated low levels of limonoid aglycones but high levels of monoterpenoids; conversely, Red-flesh Guanxi pummelo (high in carotenoids) and Guanxi pummelo (deficient in carotenoids) accumulated high levels of limonoid aglycones but low levels of monoterpenoids. However, isopentenyl diphosphate was present at similar levels. A correlation analysis indicated that jasmonic and salicylic acids might play important roles in regulating terpenoid biosynthesis. Additionally, the similarities of carotenoid and volatile profiles between each mutant and its corresponding wild type were greater than those between the two mutants or the two wild types. The flux balance of terpenoid metabolism in citrus fruit tends toward stability among various citrus genera that have different terpenoid profiles. Bud mutations could influence metabolite profiles of citrus fruit to a limited extent.
\end{abstract}

Keywords: carotenoids; aromas; bitter compounds; hormones; citrus

\section{Introduction}

In citrus, the ability to accumulate anthocyanins is not a universal feature [1]; blood orange (Citrus sinensis) and purple pummelo (Citrus maxima) are two rare citrus accessions characterized by high anthocyanin accumulation in mature fruits [1]. Usually, the main pigments of citrus fruit are carotenoids [1,2]. Fruit color is an important indicator of exterior and inner quality levels, including nutrition and flavor [3]. Usually, the juice sacs of citrus fruit are yellow or orange; however, an increase in color mutants of citrus has been reported-for example, Shara orange [4], Cara Cara navel orange [5], Red Anliu orange (R-An) [6], Chuhong pummelo [7], and Red-flesh Guanxi pummelo (R-GX) [8]. Carotenoid and primary and secondary metabolite (e.g., sugars, acids, and flavonoids) constituents in color mutants are different from those of wild types $[6,9]$.

In addition to tetraterpenoid carotenoids, monoterpenoid and sesquiterpenoid volatiles, and triterpenoid bitter compounds are important secondary metabolites of citrus fruit [7]. These terpenoids play key roles in fruit qualities, pollinator attraction, plant defense, and interactions with the surrounding environment [10]. Phytohormones of abscisic acid (ABA) and strigolactones are also synthesized by the terpenoid pathway $[11,12]$. ABA is one of the important hormones that 
regulate various aspects of fruit ripening. For example, respiratory metabolism, pigment biosynthesis, color changes, phenolic metabolism, nutritional contents, cell wall metabolism, fruit softening, and sugar and acid metabolism [13,14]. Recently, crosstalk among hormones have been studied [15,16]. Studies show that jasmonic acid (JA) and salicylic acid (SA) affect the maturation or ripening and related phenotypes of non-climacteric fruit and climacteric fruit [17-20].

Therefore, it is necessary to study the profiles and relationships of terpenoids and phytohormones in fruits of red-flesh citrus mutants. For this purpose, two red-flesh citrus mutants-R-An orange and R-GX pummelo-and their corresponding wild types were used in this study and their terpenoid profiles and constituents of phytohormones were investigated. Subsequently, the equivalent quantities of substrates for terpenoids were calculated and the effects of hormones and red-flesh bud mutations on terpenoid profiles were also revealed.

\section{Results}

In this study, two red-flesh citrus varieties and their respective wild types were used to explore the characteristics of phytohormones and terpenoid metabolites, including carotenoids, volatiles, and limonoid aglycones.

\subsection{Carotenoids}

In total, 11 carotenoids-violaxanthin, 9-Z-violaxanthin, lutein, $\alpha$-carotene, $\beta$-carotene, phytonen, antheraxanthin, zeaxanthin, $\beta$-crytoxanthin, lycopene, and $\gamma$-carotene-were detected in juice sacs (Table 1).

Table 1. Carotenoids in juice sacs of red-flesh citrus mutants and their corresponding wild types $\left(\mu \mathrm{g} / \mathrm{g} \mathrm{DW}^{\mathrm{a}}\right)$.

\begin{tabular}{|c|c|c|c|c|c|}
\hline Compounds & Code & R-An ${ }^{b}$ & An & R-GX & GX \\
\hline Violaxanthin & $\mathrm{V}$ & $4.92 \pm 0.37$ & $9.37 \pm 1.16^{*}$ & 1 & 1 \\
\hline 9-Z-Violaxanthin & $9 \mathrm{~V}$ & $8.97 \pm 0.85$ & $18.98 \pm 2.20$ * & $0.95 \pm 0.02$ & $0.86 \pm 0.01$ * \\
\hline Lutein & $\mathrm{L}$ & $0.82 \pm 0.07$ & $0.09 \pm 0.07 *$ & 1 & / \\
\hline$\alpha$-Carotene & $\mathrm{AC}$ & $0.98 \pm 0.02$ & $0.92 \pm 0.03$ & $0.89 \pm 0.02$ & $0.48 \pm 0.01$ * \\
\hline$\beta$-Carotene & $\mathrm{BC}$ & $2.21 \pm 0.19$ & $0.13 \pm 0.01 *$ & $22.21 \pm 0.19$ & Trace * \\
\hline Phytoene & $\mathrm{P}$ & / & / & $6.62 \pm 0.04$ & /* \\
\hline Antheraxanthin & A & $5.22 \pm 0.39$ & $1.45 \pm 0.30 *$ & $0.21 \pm 0.01$ & $0.42 \pm 0.05$ * \\
\hline Zeaxanthin & $\mathrm{Z}$ & / & 1 & $1.06 \pm 0.01$ & $0.81 \pm 0.01$ * \\
\hline$\beta$-Crytoxanthin & $\mathrm{CR}$ & $0.93 \pm 0.02$ & $4.39 \pm 0.37^{*}$ & l & $0.83 \pm 0.01$ * \\
\hline Lycopene & LY & $130.52 \pm 6.80$ & /* & $591.21 \pm 9.65$ & Trace * \\
\hline$\gamma$-Carotene & $\mathrm{CC}$ & l & 1 & $30.56 \pm 0.98$ & /* \\
\hline Total carotenoids & $\mathrm{TC}$ & $154.58 \pm 7.82$ & $35.34 \pm 3.84$ * & $622.55 \pm 9.13$ & $3.08 \pm 0.34$ * \\
\hline
\end{tabular}

Note: ${ }^{a}$ DW, dry weight. ${ }^{b}$ R-An, Red Anliu orange; An, Anliu orange; R-GX, Red-flesh Guanxi pummelo; GX, Guanxi pummelo; each value is the mean \pm SE; /, not detected; Trace, compounds detected at a trace level. *, significant differences between the red-flesh mutant and its corresponding wild type at $p<0.05$.

Between the color mutations and their corresponding wild types, the most distinctive differences were in the accumulated levels of lycopene and total carotenoids in the juice sacs. Lycopene in R-An and R-GX reached levels of 130.52 and $591.21 \mu \mathrm{g} / \mathrm{g}$, respectively, which accounted for $84.44 \%$ and $94.97 \%$ of total carotenoids, respectively. However, lycopene was undetected in An and only a trace level was detected in GX. In addition, the $\beta$-carotene level was significantly higher in red-flesh mutants than that in wild types. R-An and R-GX accumulated $2.21 \mu \mathrm{g} / \mathrm{g}$ and $22.21 \mu \mathrm{g} / \mathrm{g}$ of $\beta$-carotene, respectively, which represented $0.13 \mu \mathrm{g} / \mathrm{g}$ and a trace level in An and GX, respectively. Additionally, the $30.56 \mu \mathrm{g} / \mathrm{g}$ of $\gamma$-carotene accumulated in R-GX was the second highest carotenoid level, while it was undetected in the juice sacs of the other three samples. 
The levels of total carotenoids in wild types were considerably lower than in red-flesh citrus varieties. The quantity of total carotenoids in R-An was $154.58 \mu \mathrm{g} / \mathrm{g}$, which was 4.37 -fold greater than in An. For R-GX, the total carotenoid content was 622.55, which was 202.13-fold greater than in GX.

\subsection{Limonoid Aglycones}

Among juice sacs, the concentrations of limonin, nomilin, and total Limonoid aglycones (LAs) in R-An were significantly higher than those in An. However, limonin, nomilin, and total LA levels in R-GX were lower than those in GX, but only the nomilin contents were significantly different (Table 2).

Table 2. Limonoid aglycones (LAs) in juice sacs of red-flesh citrus mutants and their corresponding wild types $\left(\mu \mathrm{g} / \mathrm{g} \mathrm{DW}^{\mathrm{a}}\right)$.

\begin{tabular}{cccccc}
\hline Compounds & Code & R-An $^{\mathbf{b}}$ & An & R-GX & GX \\
\hline Limonin & LIM & $561.89 \pm 46.41$ & $449.16 \pm 8.76^{*}$ & $1439.15 \pm 153.45$ & $1722.79 \pm 200.16$ \\
Nomilin & NOM & $13.20 \pm 1.99$ & $7.02 \pm 0.29^{*}$ & $327.78 \pm 36.16$ & $426.01 \pm 17.01^{*}$ \\
Total LAs & TLA & $575.09 \pm 48.39$ & $456.18 \pm 9.03 *$ & $1766.93 \pm 186.29$ & $2148.80 \pm 189.74$ \\
\hline
\end{tabular}

Note: ${ }^{a}$ DW, dry weight. ${ }^{b}$ R-An, Red Anliu orange; An, Anliu orange; R-GX, Red-flesh Guanxi pummelo; GX, Guanxi pummelo; each value is the mean $\pm \mathrm{SE}$. *, significant gnificant differences between the red-flesh mutant and its corresponding wild type at $p<0.05$.

Importantly, the amounts of limonin, nomilin and total LAs in R-GX and GX were far greater than those in R-An and An. The concentrations of limonin in R-GX and GX were 1,439.15 and 1,722.79 $\mu \mathrm{g} / \mathrm{g}$, respectively, while those in R-An and An were 561.89 and $449.16 \mu \mathrm{g} / \mathrm{g}$, respectively. Similarly, the concentrations of nomlin in R-GX and GX were 327.78 and $426.01 \mu \mathrm{g} / \mathrm{g}$, respectively, while those in R-An and An were 13.20 and $7.02 \mu \mathrm{g} / \mathrm{g}$, respectively. Compared with 575.09 and $456.18 \mu \mathrm{g} / \mathrm{g}$ of total LAs in R-An and An, respectively, 1,766.93 and 2,148.80 $\mu \mathrm{g} / \mathrm{g}$ of total LAs were detected in R-GX and GX, respectively. On the basis of the concentrations of LAs in juice sacs, R-An and An had low-LA-containing fruit, while R-GX and GX had high-LA-containing fruit.

\subsection{Volatiles}

In the study, 71 volatiles were detected, among which 45 volatiles were positively identified with the assistance of authentic standards and 26 were tentatively identified. These volatile compounds were classified into 12 groups: 17 monoterpenes, nine monoterpene alcohols, four monoterpene aldehydes, two monoterpene esters, four monoterpene oxides, 17 sesquiterpenes, three sesquiterpene alcohols, two sesquiterpene aldehydes, one sesquiterpene ketone, one sesquiterpene oxide, four alcohols, and seven aldehydes (Table 3).

The numbers of volatiles detected in R-An, An, R-GX, and GX were 66, 65, 33, and 31, respectively. The number of volatiles with significant differences between R-An and An was 33, among which 20 volatiles had significantly higher levels in R-An than in An. Only the contents of 10 volatiles in R-GX were significantly different from in GX, among which the contents of seven volatiles were significantly higher in R-GX than in GX. Germacrene D was the unique volatile that was significantly present in greater amounts in R-An and R-GX than in their wild types. However, the contents of monoterpenes, monoterpene aldehydes, monoterpene oxides, monoterpene esters, monoterpenoids, and total volatiles in both red-flesh mutants, R-An, and R-GX, were greater than in their wild types (Table S2). 
Table 3. Volatiles in the flavedo of red-flesh citrus mutants and their corresponding wild types $\left(\mu \mathrm{g} / \mathrm{g} \mathrm{FW}^{\mathrm{a}}\right)$.

\begin{tabular}{|c|c|c|c|c|c|c|c|}
\hline Compounds ${ }^{b}$ & Code & RI $^{c}$ & RIx d & R-An ${ }^{e}$ & An & R-GX & GX \\
\hline Monoterpenes & $\mathrm{M}$ & & & & & & \\
\hline$\alpha$-Thujene ${ }^{\mathrm{T} 10}$ & M1 & 931 & 929 & $0.52 \pm 0.06$ & $0.84 \pm 0.09 *$ & / & l \\
\hline$\alpha$-Pinene & M2 & 938 & 937 & $52.34 \pm 0.18$ & $40.52 \pm 1.64$ * & $4.14 \pm 1.14$ & $3.33 \pm 0.74$ \\
\hline Camphene & M3 & 956 & 951 & $0.29 \pm 0.02$ & $0.26 \pm 0.07$ & / & l \\
\hline Sabinene & M4 & 979 & 974 & $58.70 \pm 7.11$ & $101.00 \pm 7.44^{*}$ & $3.09 \pm 0.52$ & $3.12 \pm 0.21$ \\
\hline$\beta$-Pinene & M5 & 982 & 979 & $2.98 \pm 0.33$ & $4.56 \pm 0.29 *$ & $9.56 \pm 1.24$ & $11.69 \pm 0.81$ \\
\hline$\beta$-Myrcene & M6 & 994 & 991 & $158.44 \pm 4.13$ & $115.91 \pm 5.68$ * & $243.72 \pm 82.07$ & $226.04 \pm 58.49$ \\
\hline Pseudolimonene $^{\mathrm{T} 11}$ & M7 & 1008 & 1004 & $0.31 \pm 0.05$ & $0.13 \pm 0.12$ & 1 & 1 \\
\hline$\alpha$-Phellandrene & M8 & 1011 & 1005 & $13.10 \pm 2.38$ & $13.71 \pm 1.40$ & $0.14 \pm 0.25$ & / \\
\hline$\alpha$-Terpinene & M9 & 1023 & 1017 & $0.37 \pm 0.07$ & $0.44 \pm 0.04$ & / & / \\
\hline Sylvestrene $\mathrm{e}^{\mathrm{T} 11}$ & M10 & 1027 & 1027 & $0.15 \pm 0.13$ & $0.24 \pm 0.09$ & / & / \\
\hline d-Limonene & M11 & 1038 & 1030 & $7846.10 \pm 436.16$ & $6647.90 \pm 347.94$ * & $878.30 \pm 282.27$ & $656.70 \pm 183.73$ \\
\hline$\beta$-Phellandrene $\mathrm{T}^{\mathrm{T} 10}$ & M12 & 1040 & 1031 & $0.60 \pm 0.25$ & $1.48 \pm 0.16^{*}$ & / & / \\
\hline$(Z)$ - $\beta$-Ocimene & M13 & 1044 & 1038 & $0.54 \pm 0.04$ & $0.43 \pm 0.11$ & $0.26 \pm 0.01$ & $0.24 \pm 0.05$ \\
\hline (E)- $\beta$-Ocimene & M14 & 1055 & 1049 & $10.88 \pm 1.07$ & $9.20 \pm 1.52$ & $7.22 \pm 0.18$ & $5.51 \pm 0.80$ * \\
\hline 4-Carene $\mathrm{e}^{\mathrm{T} 11}$ & M15 & 1060 & 1009 & $0.33 \pm 0.07$ & $1^{*}$ & / & / \\
\hline$\gamma$-Terpiene & M16 & 1066 & 1060 & $0.52 \pm 0.09$ & $0.62 \pm 0.01$ & l & l \\
\hline Terpinolene & M17 & 1090 & 1088 & $3.50 \pm 0.69$ & $3.69 \pm 0.33$ & $0.23 \pm 0.02$ & $0.12 \pm 0.11$ \\
\hline Monoterpene Alcohols & MA & & & & & & \\
\hline (Z)-Sabinene hydrate & MA1 & 1079 & 1077 & $2.53 \pm 0.25$ & $3.28 \pm 0.41$ & l & l \\
\hline$\beta$-Linalool & MA2 & 1107 & 1099 & $107.93 \pm 4.81$ & $38.65 \pm 3.79 *$ & $2.67 \pm 0.92$ & $2.57 \pm 0.15$ \\
\hline (E)-p-Mentha-2,8-dienol ${ }^{\mathrm{T} 12}$ & MA3 & 1133 & 1123 & $0.49 \pm 0.05$ & $0.24 \pm 0.04 *$ & 1 & 1 \\
\hline 4-Terpineol & MA4 & 1190 & 1177 & $0.65 \pm 0.02$ & $0.67 \pm 0.10$ & i & / \\
\hline$\alpha$-Terpineol & MA5 & 1206 & 1189 & $10.70 \pm 0.65$ & $7.65 \pm 1.03 *$ & $1.15 \pm 0.43$ & $1.24 \pm 0.25$ \\
\hline (E)-Piperitol ${ }^{\mathrm{T} 1}$ & MA6 & 1220 & 1208 & 1 & 1 & $0.14 \pm 0.12$ & $0.12 \pm 0.11$ \\
\hline Citronellol & MA7 & 1236 & 1228 & $1.55 \pm 0.13$ & $1.66 \pm 0.25$ & / & / \\
\hline Geraniol & MA8 & 1261 & 1255 & $2.03 \pm 0.26$ & $2.47 \pm 0.54$ & $1.47 \pm 0.77$ & $2.01 \pm 0.25$ \\
\hline p-Mentha-1(7),8(10)-dien-9-ol ${ }^{\mathrm{T} 1}$ & MA9 & 1303 & / & $1.68 \pm 0.10$ & $0.82 \pm 0.15$ * & 1 & 1 \\
\hline Monoterpene alDehydes & MD & & & & & & \\
\hline Citronellal & MD1 & 1164 & 1153 & $7.85 \pm 0.38$ & $5.27 \pm 0.25 *$ & / & l \\
\hline Neral & MD2 & 1251 & 1240 & $16.22 \pm 0.58$ & $12.01 \pm 0.81$ * & $2.28 \pm 0.77$ & $1.56 \pm 0.54$ \\
\hline Geranial & MD3 & 1281 & 1270 & $22.28 \pm 0.70$ & $16.47 \pm 1.60$ * & $2.87 \pm 1.02$ & $2.22 \pm 0.83$ \\
\hline Perillal $^{\mathrm{T} 13}$ & MD4 & 1291 & 1272 & $6.44 \pm 0.27$ & $2.49 \pm 0.20 *$ & 1 & / \\
\hline Monoterpene Esters & $\mathrm{ME}$ & & & & & & \\
\hline
\end{tabular}


Table 3. Cont.

\begin{tabular}{|c|c|c|c|c|c|c|c|}
\hline Compounds ${ }^{b}$ & Code & $\mathrm{RI}^{\mathrm{c}}$ & $\operatorname{RIx}^{\mathrm{d}}$ & R-An ${ }^{e}$ & An & R-GX & GX \\
\hline Neryl acetate & ME1 & 1366 & 1364 & $0.46 \pm 0.06$ & $0.30 \pm 0.07$ * & $0.26 \pm 0.04$ & $1^{*}$ \\
\hline Geranyl acetate & ME2 & 1385 & 1382 & $0.32 \pm 0.28$ & $0.27 \pm 0.14$ & $0.57 \pm 0.08$ & $0.26 \pm 0.06^{*}$ \\
\hline Monoterpene Oxides & $\mathrm{MO}$ & & & & & & \\
\hline (Z)-Linalool oxide & MO1 & 1078 & 1074 & / & / & $0.30 \pm 0.07$ & $0.09 \pm 0.02 *$ \\
\hline (E)-Linalool oxide & $\mathrm{MO} 2$ & 1093 & 1086 & 1 & / & $0.16 \pm 0.14$ & 1 \\
\hline (Z)-Limonene oxide & $\mathrm{MO} 3$ & 1143 & 1134 & $0.46 \pm 0.04$ & $0.39 \pm 0.07$ & 1 & 1 \\
\hline (E)-Limonene oxide & $\mathrm{MO} 4$ & 1148 & 1138 & $3.24 \pm 0.09$ & $0.21 \pm 0.05 *$ & $0.29 \pm 0.07$ & $0.27 \pm 0.09$ \\
\hline Sesquiterpenes & S & & & & & & \\
\hline$\delta$-Elemene ${ }^{\mathrm{T} 13}$ & S1 & 1338 & 1338 & l & / & $1.95 \pm 0.58$ & $1.64 \pm 0.14$ \\
\hline Copaene $\mathrm{T}^{\mathrm{T} 13}$ & S2 & 1380 & 1376 & $4.18 \pm 0.37$ & $2.26 \pm 0.13 *$ & / & / \\
\hline$\beta$-Cubebene ${ }^{\mathrm{T} 3}$ & S3 & 1391 & 1389 & $3.13 \pm 0.18$ & $1.59 \pm 0.07 *$ & 1 & 1 \\
\hline$\beta$-Elemene ${ }^{\mathrm{T} 13}$ & S4 & 1393 & 1391 & $1.17 \pm 0.10$ & $1.14 \pm 0.05$ & $0.93 \pm 0.29$ & $0.93 \pm 0.17$ \\
\hline Caryophyllene & S5 & 1424 & 1419 & $2.88 \pm 0.10$ & $6.55 \pm 0.09 *$ & $1.72 \pm 0.22$ & $0.75 \pm 0.28$ * \\
\hline$\beta$-Farnesene & S6 & 1457 & 1457 & $0.84 \pm 0.10$ & $5.05 \pm 0.29 *$ & / & / \\
\hline$\alpha$-Caryophyllene & S7 & 1461 & 1454 & $0.67 \pm 0.03$ & $0.50 \pm 0.02 *$ & / & / \\
\hline Germacrene $\mathrm{D}^{\mathrm{T} 2}$ & S8 & 1487 & 1481 & $2.85 \pm 0.18$ & $1.64 \pm 0.08 *$ & $11.59 \pm 1.98$ & $7.11 \pm 2.05^{*}$ \\
\hline$\gamma$-Selinene ${ }^{\mathrm{T} 3}$ & S9 & 1489 & 1481 & $0.41 \pm 0.05$ & $0.79 \pm 0.16^{*}$ & 1 & 1 \\
\hline Valencene & S10 & 1497 & 1492 & $8.89 \pm 0.11$ & $16.19 \pm 0.62 *$ & $2.34 \pm 0.34$ & $2.37 \pm 0.52$ \\
\hline Elixene ${ }^{\mathrm{T} 13}$ & S11 & 1500 & 1471 & $1.38 \pm 0.05$ & $1.10 \pm 0.40$ & $1.70 \pm 0.20$ & $1.22 \pm 0.20$ * \\
\hline$\alpha$-Muurolene ${ }^{\mathrm{T} 3}$ & $\mathrm{~S} 12$ & 1505 & 1499 & $0.62 \pm 0.12$ & $0.50 \pm 0.09$ & 1 & 1 \\
\hline$\alpha$-Farnesene ${ }^{\mathrm{T} 4}$ & S13 & 1508 & 1508 & $1.11 \pm 0.16$ & $12.86 \pm 1.42 *$ & / & / \\
\hline$\delta$-Cadinene ${ }^{\mathrm{T} 13}$ & S14 & 1524 & 1524 & $4.91 \pm 0.40$ & $2.43 \pm 0.20 *$ & 1 & 1 \\
\hline$\alpha$-Panasinsen $\mathrm{T} 13$ & S15 & 1525 & 1527 & $0.13 \pm 0.22$ & $0.69 \pm 0.15 *$ & $0.50 \pm 0.13$ & $0.60 \pm 0.18$ \\
\hline$\beta$-Sesquiphellandrene ${ }^{\mathrm{T} 4}$ & S16 & 1530 & 1524 & $0.27 \pm 0.06$ & $0.46 \pm 0.12$ & 1 & 1 \\
\hline Germacrene B T13 & S17 & 1566 & 1557 & / & / & $0.45 \pm 0.39$ & $0.49 \pm 0.16$ \\
\hline Sesquiterpene aLcohols & SL & & & & & & \\
\hline$(E)$-Nerolidol & SL1 & 1567 & 1564 & $0.99 \pm 0.12$ & $0.73 \pm 0.16$ & / & / \\
\hline Germacrene D-4-ol ${ }^{\mathrm{T} 3}$ & SL2 & 1585 & 1574 & $0.43 \pm 0.03$ & $0.51 \pm 0.11$ & / & / \\
\hline Farnesol & SL3 & 1727 & 1713 & $1.02 \pm 0.09$ & $0.89 \pm 0.23$ & $0.73 \pm 0.66$ & $4.82 \pm 0.40$ * \\
\hline Sesquiterpene alDehydes & SD & & & & & & \\
\hline$\beta$-Sinensal ${ }^{\mathrm{T} 3}$ & SD1 & 1705 & 1695 & $1.31 \pm 0.16$ & $1.04 \pm 0.43$ & / & / \\
\hline$\alpha$-Sinensal ${ }^{\mathrm{T} 3}$ & SD2 & 1767 & 1752 & $1.39 \pm 0.53$ & $2.46 \pm 0.71$ & / & / \\
\hline Sesquiterpene Ketone & SK & & & & & & \\
\hline Nootkatone & SK1 & 1824 & 1808 & $0.69 \pm 0.12$ & $1.47 \pm 0.56$ & / & / \\
\hline Sesquiterpene oxide & $\mathrm{SO}$ & & & & & & \\
\hline
\end{tabular}


Table 3. Cont.

\begin{tabular}{|c|c|c|c|c|c|c|c|}
\hline Compounds $b$ & Code & $\mathrm{RI}^{\mathrm{c}}$ & $\operatorname{RIx}^{d}$ & R-An ${ }^{e}$ & An & R-GX & GX \\
\hline Caryophyllene oxide & $\mathrm{SO} 1$ & 1591 & 1581 & $0.58 \pm 0.11$ & $1.86 \pm 0.31 *$ & 1 & 1 \\
\hline $\begin{array}{l}\text { ALcohols } \\
\text { (Z)-3-Hexenol }\end{array}$ & $\begin{array}{l}\text { AL } \\
\text { AL1 }\end{array}$ & 868 & 856 & $2.35 \pm 0.34$ & $2.36 \pm 0.20$ & $0.70 \pm 0.09$ & $0.73 \pm 0.12$ \\
\hline (E)-2-Hexenol & AL2 & 878 & 862 & 1 & 1 & 1 & $0.49 \pm 0.12$ * \\
\hline Hexanol & AL3 & 881 & 868 & $0.94 \pm 0.31$ & $1.04 \pm 0.06$ & $0.06 \pm 0.10$ & $0.35 \pm 0.08 *$ \\
\hline Octanol & AL4 & 1081 & 1071 & $3.65 \pm 1.01$ & $2.91 \pm 0.64$ & 1 & / \\
\hline AlDehydes & $\mathrm{AD}$ & & & & & & \\
\hline 3-Hexenal ${ }^{\mathrm{T} 7}$ & AD1 & 811 & 810 & $0.56 \pm 0.40$ & $0.67 \pm 0.12$ & / & / \\
\hline Hexanal & $\mathrm{AD} 2$ & 812 & 800 & $2.06 \pm 0.59$ & $1.76 \pm 0.31$ & $0.10 \pm 0.17$ & $0.21 \pm 0.19$ \\
\hline (E)-2-Hexenal & AD3 & 870 & 854 & $1.09 \pm 0.14$ & $0.71 \pm 0.15$ * & 1 & 1 \\
\hline Nonanal & $\mathrm{AD} 4$ & 1116 & 1104 & $2.62 \pm 0.57$ & $1.62 \pm 0.22 *$ & / & 1 \\
\hline Decanal & AD5 & 1216 & 1206 & $12.84 \pm 3.15$ & $8.99 \pm 1.73$ & $0.33 \pm 0.12$ & $0.42 \pm 0.05$ \\
\hline Undecanal & AD6 & 1318 & 1307 & $0.88 \pm 0.12$ & $0.42 \pm 0.11 *$ & / & l \\
\hline Dodecanal & AD7 & 1418 & 1409 & $2.34 \pm 0.52$ & $1.61 \pm 0.15$ & l & / \\
\hline Monoterpenes & $\mathrm{TM}$ & & & 8149.68 & 6940.94 & 1146.67 & 906.74 \\
\hline Monoterpene Alcohols & TMA & & & 127.57 & 55.45 & 5.43 & 5.93 \\
\hline Monoterpene alDehydes & TMD & & & 52.78 & 36.23 & 5.14 & 3.78 \\
\hline Monoterpene Oxides & TMO & & & 3.70 & 0.60 & 0.75 & 0.36 \\
\hline Monoterpene Esters & TME & & & 0.78 & 0.57 & 0.83 & 0.26 \\
\hline Sesquiterpenes & TS & & & 33.40 & 53.74 & 21.17 & 15.12 \\
\hline Sesquiterpene aLcohols & TSL & & & 2.43 & 2.13 & 0.73 & 4.82 \\
\hline Sesquiterpene alDehydes & TSD & & & 2.70 & 3.49 & / & l \\
\hline Sesquiterpene Ketone & TSK & & & 0.69 & 1.47 & / & / \\
\hline Sesquiterpene Oxide & TSO & & & 0.58 & 1.86 & / & / \\
\hline Alcohols & TA & & & 6.94 & 6.31 & 0.76 & 1.57 \\
\hline AlDehydes & TAD & & & 22.38 & 15.79 & 0.43 & 0.64 \\
\hline MonoTerpenoids & TMT & & & 8334.51 & 7033.79 & 1158.82 & 917.08 \\
\hline SesquiTerpenoids & TST & & & 39.80 & 62.70 & 21.90 & 19.93 \\
\hline Non-Terpenoids & TNT & & & 29.33 & 22.10 & 1.19 & 2.21 \\
\hline Total Volatiles & TV & & & 8403.64 & 7118.58 & 1181.91 & 939.22 \\
\hline
\end{tabular}

Note: ${ }^{a}$ FW, fresh weight. ${ }^{\mathrm{b}}$ Tn, quantified by total ion current mode, while unlabeled compounds were quantified by selective ion monitoring mode, according to Table 1 in Liu et al. [21] (Table S1). ${ }^{c}$ RI, retention index on a TR-5 column in the study. ${ }^{d}$ RIx, retention index on a semistandard nonpolar column. Values were obtained from the NIST 2014 library

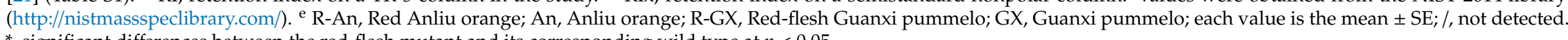
*, significant differences between the red-flesh mutant and its corresponding wild type at $p<0.05$. 
Additionally, the total volatile contents of R-An and An were 8,403.64 and 7,118.58 $\mu \mathrm{g} / \mathrm{g}$, which were far greater than in R-GX $(1181.91 \mu \mathrm{g} / \mathrm{g})$ and GX $(939.22 \mu \mathrm{g} / \mathrm{g})$, which was mainly caused by a sharp decrease in d-limonene contents in R-GX and GX. The contents of d-limonene in R-An and An were 7846.10 and $6647.90 \mu \mathrm{g} / \mathrm{g}$, respectively, while in R-GX and GX the contents were 878.30 and $656.70 \mu \mathrm{g} / \mathrm{g}$, respectively (Table 3). Correspondingly, the percentages of d-limonene in R-GX and GX were $74.31 \%$ and $69.92 \%$, respectively, while the values were $93.37 \%$ and $93.39 \%$ in R-An and An, respectively. However, the percentages of total monoterpenes in four citrus varieties were very similar, ranging from $96.54 \%$ in GX to $97.50 \%$ in An, which was caused by increases in the $\beta$-myrcene contents in R-GX and GX. The contents of $\beta$-myrcene in R-GX and GX were 243.72 and $226.04 \mu \mathrm{g} / \mathrm{g}$, respectively, but the $\beta$-myrcene contents were 158.44 and $15.91 \mu \mathrm{g} / \mathrm{g}$ in R-An and An, respectively. As a result, the quantities of $d$-limonene and $\beta$-myrcene increased to $93.99-95.25 \%$ in the four citrus varieties. Additionally, monoterpene alcohols, monoterpene aldehydes, sesquiterpenes, and monoterpenes were also the main volatiles in citrus fruit (Figure S1).

Thus, the percentages of monoterpenes in the citrus fruit used in the study were similar, while the absolute amounts of total monoterpenes were very different. According to the total monoterpene contents, R-An and An had high-level monoterpene-containing fruit, while R-GX and GX were low-level monoterpene-containing fruit.

\subsection{Isopentenyl Diphosphate (IPP) Content Analysis}

In this study, odor-related monoterpenoid and sesquiterpenoid volatiles, bitter limonoid aglycones of triterpenoids, and color-related carotenoids of tetraterpenoids were identified. IPP and dimethylallyl diphosphate, as their common precursors, could be interconverted with the assistance of isopentenyl diphosphate delta-isomerase in plastids. Therefore, the IPP contents were calculated based on quantities of various terpenoids. As shown in Figure 1, the total IPP contents ranged from 14,811.39 $\mu \mathrm{g} / \mathrm{g}$ in GX to 21,474.96 $\mu \mathrm{g} / \mathrm{g}$ in R-An, which were similar. However, the total IPPs of R-An and An were dominated by IPP converted from monoterpenoids, whereas the total IPPs of R-GX and GX were dominated by IPP converted from LAs. A partial correlation analysis showed that the correlation coefficient between total monoterpenoids and total LAs was $-0.97(p<0.05)$. This indicated that a general balance and a stable metabolic flux in the terpenoid biosynthetic pathway were maintained in citrus fruit.

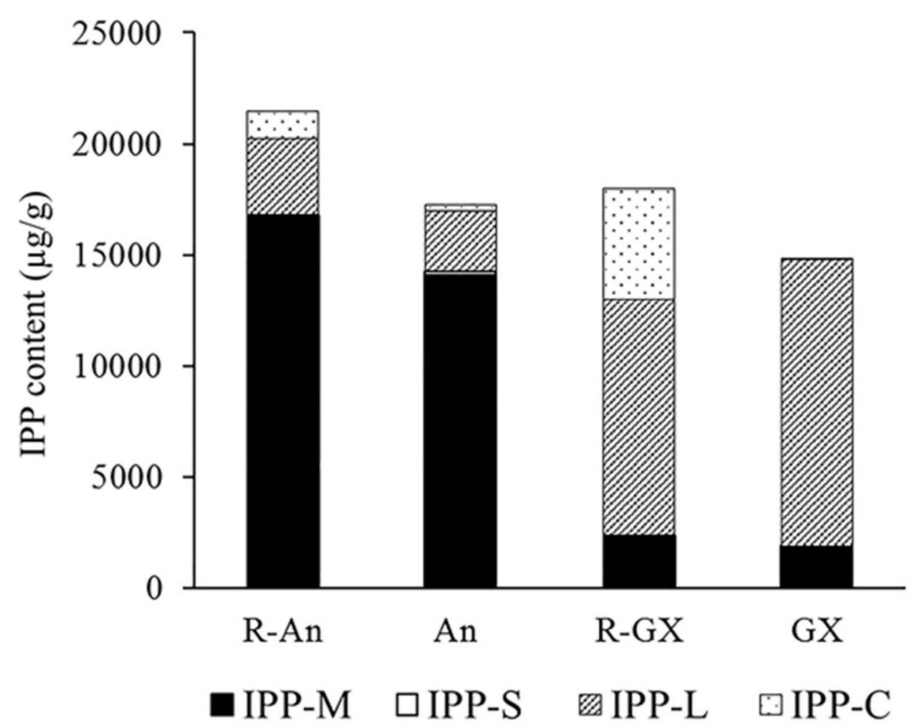

Figure 1. Total quantities of isopentenyl diphosphates (IPPs) used as substrates by four groups of terpenoids $(\mu \mathrm{g} / \mathrm{g})$. IPP-M, the quantities of IPPs converted from total monoterpenoids multiplied by 2; IPP-S, the quantities of IPPs converted from total sesquiterpenoids multiplied by 3; IPP-L, the quantities 
of IPPs converted from total limonoid aglycones multiplied by 6; IPP-C, the quantities of IPPs converted from total carotenoids multiplied by 8. R-An, Red Anliu orange; An, Anliu orange; R-GX, Red-flesh Guanxi pummelo; GX, Guanxi pummelo.

\subsection{Phytohormones in Citrus Fruits}

In the juice sacs of citrus fruit used in this study, four phytohormones were detected, ABA, IAA, $\mathrm{JA}$, and SA. Interestingly, the ABA contents in both red-flesh mutants were significantly lower than those in their corresponding wild types, respectively (Table 4). ABA dominated the phytohormone profiles of all the juice sac samples used in the studies, with the ABA contents ranging from $97.40 \%$ in R-GX to $97.82 \%$ in GX.

IAA was only detected in An at $17.35 \pm 0.51 \mathrm{ng} / \mathrm{kg}$. No significant difference was detected in the JA contents between R-An and An, while it was significantly higher in R-GX than in GX. The SA concentrations in the juice sacs ranged from $12.24 \pm 2.85 \mathrm{ng} / \mathrm{kg}$ in An to $19.38 \pm 6.82 \mathrm{ng} / \mathrm{kg}$ in GX; no significant differences were detected between either R-An and An or R-GX and GX (Table 4).

Table 4. Phytohormones in juice sacs of red-flesh citrus mutants and their corresponding wild types $\left(\mathrm{ng} / \mathrm{gDW}^{\mathrm{a}}\right)$.

\begin{tabular}{ccccc}
\hline & R-An $^{\mathbf{b}}$ & An & R-GX & GX \\
\hline ABA & $1428.49 \pm 103.87$ & $2242.52 \pm 126.82^{*}$ & $1089.35 \pm 50.03$ & $1276.14 \pm 53.30^{*}$ \\
IAA & $/$ & $17.35 \pm 0.51^{*}$ & $/$ & $/$ \\
JA & $19.01 \pm 4.32$ & $23.02 \pm 7.43$ & $9.90 \pm 0.38$ & $9.04 \pm 0.24^{*}$ \\
SA & $13.28 \pm 2.09$ & $12.24 \pm 2.85$ & $19.12 \pm 3.01$ & $19.38 \pm 6.82$ \\
\hline
\end{tabular}

Note: ${ }^{\text {a }} \mathrm{DW}$, dry weight. ${ }^{\mathrm{b}}$ R-An, Red Anliu orange; An, Anliu orange; R-GX, Red-flesh Guanxi pummelo; GX, Guanxi pummelo; each value is the mean $\pm \mathrm{SE}$; /, not detected. *, significant differences between the red-flesh mutant and its corresponding wild type at $p<0.05$.

\subsection{The Correlation Between Plant Hormones and Terpenoids}

The correlation matrix was calculated based on the levels of 29 terpenoids and three hormones that appeared in all the samples used in the study. As shown in Figure 2, ABA was positively correlated with 9-Z-violaxanthin and valencene. JA was negatively correlated with $\beta$-myrcene, limonin, and nomilin, and positively correlated with 9-Z-violaxanthin, valencene, sabinene, and terpinolene. SA was positively correlated with $\beta$-myrcene, limonin and nomilin, and negatively correlated with sabinene, terpinolene, $\beta$-elemene, $\alpha$-pinene, and d-limonene. This indicated that JA and SA might be important hormones for regulating terpenoid metabolism in citrus fruit.

\subsection{Principal Component Analysis Based on Carotenoids and Volatiles}

To characterize the red-flesh mutants and their corresponding wild types, a principal component analysis (PCA) was employed using the carotenoids (Figure 3A) and volatile compounds (Figure 3B). As shown in Figure 3, the first two components in Figure 3A,B explained up to 83.0\% and 86.8\% of the variance, respectively. The four citrus fruits were completely separated in the PCA plots. Importantly, R-An and An, and R-GX and GX, could be separated on the PC1 axis, explaining 59.6\% and $74.4 \%$ of the variance in Figure $3 \mathrm{~A}, \mathrm{~B}$, respectively. Additionally, the heatmap dendrogram analysis also suggested that R-An and An clustered together, as did R-GX and GX (Figure S2). Furthermore, both red-mutants (R-An and R-GX) and both wild types (An and GX) were separated clearly on the PC2 axis, explaining $23.4 \%$ and $12.4 \%$ of the variance in Figure $3 \mathrm{~A}, \mathrm{~B}$, respectively. Their loading plots are shown in Figure S3. 


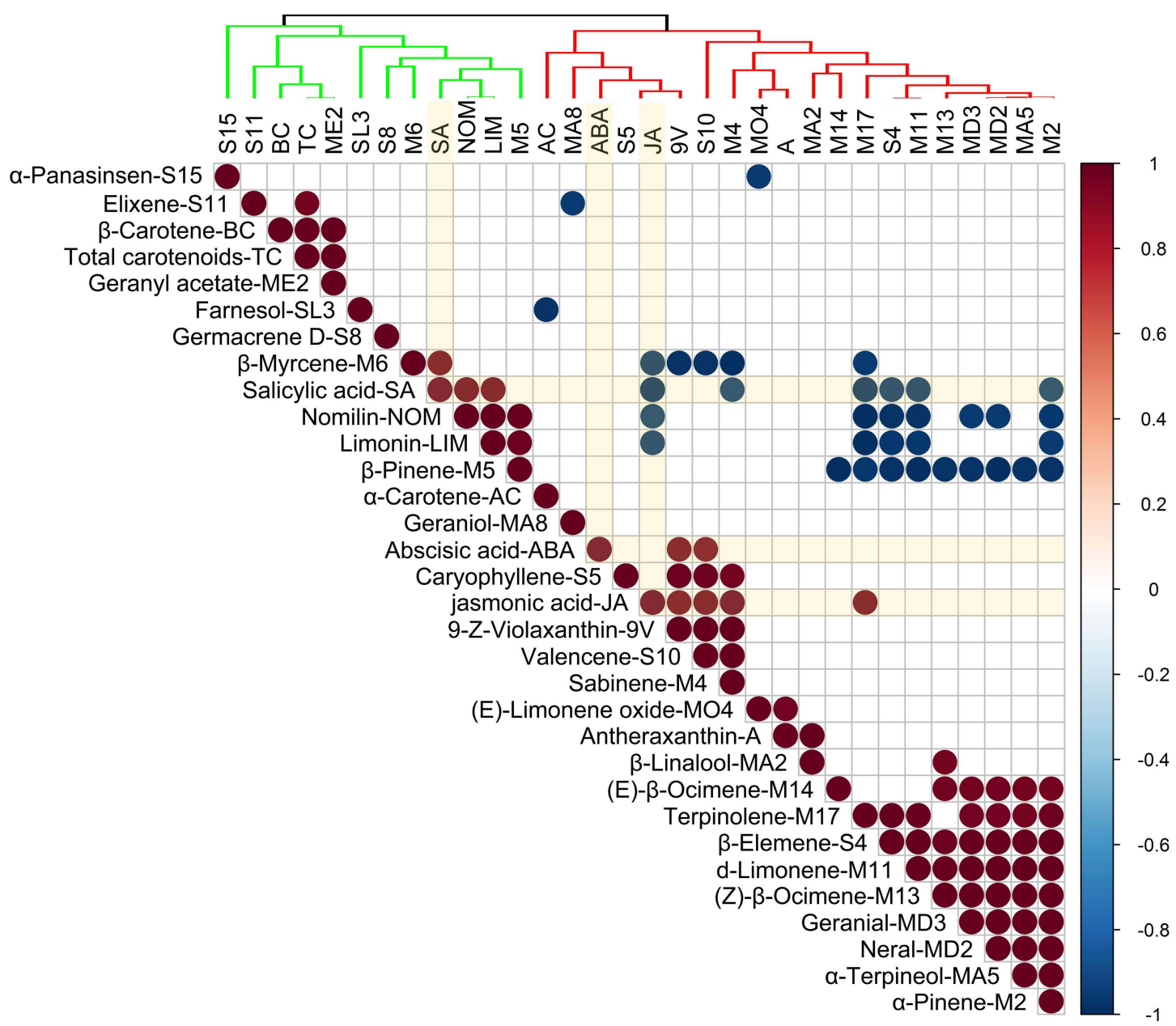

Figure 2. Correlation analysis using the main compounds in this study.

A

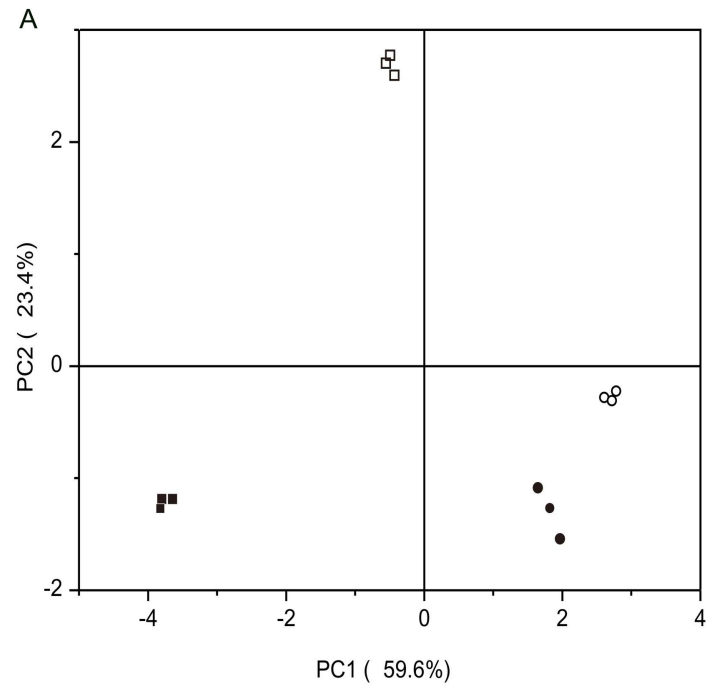

- R-An

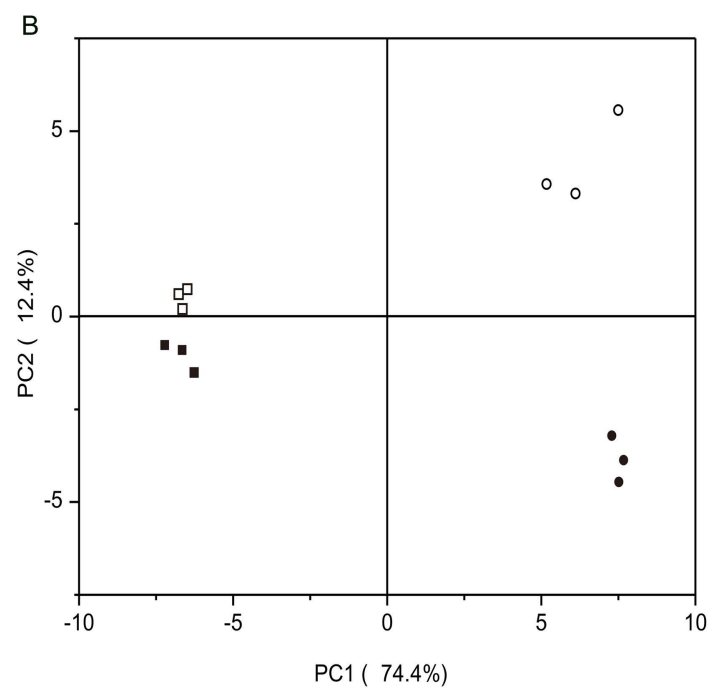

- R-GX

GX

Figure 3. Principal component analysis (PCA) score plot. (A) PCA score plot of carotenoids; (B) PCA score plot of volatile compounds. R-An, Red Anliu orange; An, Anliu orange; R-GX, Red-flesh Guanxi pummelo; GX, Guanxi pummelo 


\section{Discussion}

\subsection{Equivalent Quantities of IPP in the Terpenoid Biosynthetic Pathway Are Prone to Stability}

Two different citrus species of fruits, sweet oranges of R-An and An (Citrus sinensis), together with pummelos of R-GX and GX (Citrus maxima), were employed in the study and their terpenoid profiles were very different. Both R-An (with a high carotenoid content) and An (with a low carotenoid content) accumulated low levels of LAs but high levels of monoterpenoids; conversely, R-GX (with a high carotenoid content) and GX (which was deficient in carotenoids) accumulated high levels of LAs but low levels of monoterpenoids. However, their levels of equivalent quantities of IPP, the common substrate of terpenoids, were similar; that is, the roughly similar amounts of isoprene carbon were produced by them. This suggested that the flux balance of terpenoid metabolism in citrus fruit was prone to stability among various citrus genera that had different terpenoid profiles, and the variation of terpenoid profiles among these citrus genera was caused by the partitioning of carbon in downstream isoprenoid pathways but not increased flux through the isoprenoid pathway, which agreed with previous studies. Liu et al. [7] found a total capacity or a balance in the production of various terpenoids in pummelo. Li et al. [22] revealed that the plastid methylerythritol phosphate pathway was enhanced in Niurouhong tangerine (Citrus reticulata Blanco), which has a beef-red color, resulting from a great accumulation of $\beta$-cryptoxanthin and $\beta$-carotene, and compared with its wild type, the cytosolic mevalonic acid pathway was suppressed in Niurouhong tangerine. Recent developments are predominantly driven by experimental and computational advancements, producing a network-wide metabolic flux and metabolome maps, which enable the determination of cellular metabolism and the estimation of metabolic flux in vivo by applying a natural isotope correction of the MS/MS measurements [23]. Wada et al. [24] constructed a mevalonic acid (MVA)-producing strain of Escherichia coli by introducing acetoacetyl-CoA synthase/3-hydroxy-3-methylglutaryl-CoA (HMG-CoA) reductase and HMG-CoA synthase genes from Enterococcus faecalis. The ${ }^{13} \mathrm{C}$-metabolic flux analysis revealed that the MVA yield of the engineered strain was close to the upper limit, but the flux levels in acetate formation and the TCA cycle in the engineered strain were lower than those in the control strain. This indicated that the metabolic flux levels in these organisms are prone to stability.

\subsection{Effects of Hormones on Terpenoid Metabolism}

Among the four hormones investigated in the study, only the ABA contents of both red-flesh mutants were significantly lower than those in wild types, which agreed with the results in Niurouhong tangerine (Citrus reticulata Blanco) and Pinalate (Citrus sinensis L. Osbeck), two previously reported citrus color mutants [22,25]. A significant decrease in ABA-a downstream metabolite of the carotenoid biosynthetic pathway-in mutants that had high levels of some carotenoids was very common, and the mechanism should be further studied.

JA and SA were significantly correlated with a variety of terpenoids, which indicated that JA and SA might be important hormones involved in regulating terpenoid biosynthesis in citrus fruit. In Panax ginseng adventitious roots treated with SA, the contents of JA and some terpenoids-such as farensol, isochiapin B sesquiterpenoids, champhor, and cineole monoterpenoids-are higher [26]. SA and terpenoid volatiles, including (E)- $\beta$-ocimene, linalool, methyl salicylate, indole, caryophyllene, $\beta$-elemene, and $\alpha$-farnesene, are accumulated in tobacco plants infected with the avirulent strain Pseudomonas syringae pv. Maculicola ES4326 or pv. Tomato DC3000 [27]. The biosynthesis of (E)- $\beta$-ocimene, linalool, $\beta$-caryophyllene, and germacrene $\mathrm{D}$ can be induced by methyl jasmonate in grape leaf [28]. Similar results were also reported in sweet basil, tomato, and Catharanthus roseus [29-32]. The biosynthetic and regulatory mechanisms of terpenoids in citrus should be further studied.

\subsection{Effects of Red-Flesh Bud Mutations on Metabolite Profiles}

Both the R-An and R-GX red-flesh mutants used in the study were spontaneous bud mutations [6,8]. Compared with wild types, multiple metabolite profiles of red-flesh mutants varied, 
including carotenoids, volatiles, bitter compounds, hormones, flavonoids, sugars, and acids in this study and previous studies [6-9,22,25]. Additionally, the similarities in carotenoid and volatile profiles between each mutant and its corresponding wild type were greater than those between both mutants or both wild types (Figure 3 and Figure S2), which suggested that bud mutations could influence metabolite profiles of citrus fruit to some extent, but the influence was limited. Notably, R-An and R-GX highly accumulate lycopene in their fruit pulp, which demonstrate distinctive red-flesh and have high economic and health value. Therefore, these red-flesh mutants are more popular than their wild types by planters and consumers. Especially, R-GX accumulated considerably high contents of carotenoids and LAs, which have been reported to be responsible for a chemopreventive and therapeutic role in human health, as demonstrated in large cohort and case control studies of cancer, heart disease, and many other diseases [33]. It is expected that R-An and R-GX will be used more widely than their wild types for table fruits or red-fleshed citrus juices as potential natural resources because of their higher nutritional and medicinal properties [34].

\section{Materials and Methods}

\subsection{Plant Materials}

Mature fruits of R-An and Anliu (An) orange (Citrus sinensis) and R-GX and Guanxi pummelo (GX) (Citrus maxima) were harvested in the winter of 2011. Detailed fruit sample information is listed in Table 5.

The sample preparations for flavedo and juice sacs were conducted in accordance with Liu et al. $[7,21]$. The flavedo around the equatorial plane of fruits was snap-frozen in liquid nitrogen without touching the inner part of the fruit. A portion of the flavedo was stored at $-80{ }^{\circ} \mathrm{C}$ for further volatile compound extractions and analysis. The juice sacs were lyophilized (Heto Lyolab 3000, Heto-Holten A/S, Allerød, Denmark) and stored at $-80{ }^{\circ} \mathrm{C}$ for carotenoid, bitter compound, and hormone extractions. Three independent biological replicates were prepared for each sample.

Table 5. Sampling information for fruits of red-flesh citrus mutants and their corresponding wild types.

\begin{tabular}{|c|c|c|c|c|}
\hline Cultivar & Flesh Color & Code & Production Area & Remarks \\
\hline $\begin{array}{l}\text { Red Anliu orange } \\
\text { Anliu orange }\end{array}$ & $\begin{array}{l}\text { Red flesh } \\
\text { Yellow flesh }\end{array}$ & $\begin{array}{l}\text { R-An } \\
\text { An }\end{array}$ & Citrus Research Institute of Guangxi, & $\begin{array}{l}\text { Red-flesh mutant of Anliu orange } \\
\text { Common sweet orange }\end{array}$ \\
\hline $\begin{array}{l}\text { Red-flesh Guanxi pummelo } \\
\text { Guanxi pummelo }\end{array}$ & $\begin{array}{c}\text { Red flesh } \\
\text { Pale yellow flesh }\end{array}$ & $\begin{array}{l}\text { R-GX } \\
\text { GX }\end{array}$ & $\begin{array}{c}\text { Fujian Academy of Agricultural Sciences, } \\
\text { Xiamen, Fujian province }\end{array}$ & $\begin{array}{c}\text { Red-flesh mutant } \\
\text { of Guanxi pummelo } \\
\text { Common white-flesh pummelo }\end{array}$ \\
\hline
\end{tabular}

\subsection{Standards and Reagents}

For the carotenoid analysis, antheraxanthin, $\alpha$-carotene, $\beta$-cryptoxanthin, all-trans-lutein, phytoene, and all-trans-violaxanthin were obtained commercially from CaroteNature (Lupsingen, Switzerland); $\beta$-carotene and all-trans-lycopene were obtained from Sigma Co. Ltd. (St Louis, MO, USA).

For the bitter compound analysis, limonin, nomilin, and naringin of high-performance liquid chromatography (HPLC) grade were purchased from Sigma Co. Ltd.

For the plant hormone analysis, indol-3-acetic-2,2- $\left[{ }^{2} \mathrm{H}_{5}\right]$ acid $\left(\left[{ }^{2} \mathrm{H}_{5}\right] \mathrm{IAA}\right)$, indole-3-actic acid (IAA), 2-hydroxy-benzoic acid- $\left[{ }^{2} \mathrm{H}_{4}\right]\left(\left[{ }^{2} \mathrm{H}_{4}\right] \mathrm{SA}\right), \mathrm{SA}, 3-$ oxo-2-(cis-2-pentenyl) cyclopentane-1- $\left[{ }^{2} \mathrm{H}_{2}\right]$ acetic acid $\left(\left[{ }^{2} \mathrm{H}_{2}\right] J A\right), J A, 2-c i s, 4-t r a n s-a b s c i s i c ~ a c i d-\left[{ }^{2} \mathrm{H}_{6}\right]\left(\left[{ }^{2} \mathrm{H}_{6}\right] \mathrm{ABA}\right)$, and ABA were purchased from OlChemIm Ltd. (Olomouc, Czech Republic).

For the volatile analysis, the internal standard of methyl nonanoate was obtained from Sigma Co. Ltd. A standard series of $C_{7}-C_{30}$ saturated alkanes from Supelco (Bellefonte, PA., USA) were used for retention index (RI) determination. The sources of the volatile standards are listed in Table 1 of a previously published paper (Table S1) [21].

In addition, the following were used in the metabolite extraction solution: methyl tertbutyl ether (MTBE), methol, and acetonitrile (HPLC grade) from Tedia (Fairfield, CT, USA); and triethylamine 
(HPLC grade), ethanol, hexane, acetone, iso-propyl alcohol, dichloromethane, trichloromethane, and methanoic acid from Sinopharm Chemical Reagent Co., Ltd. (Shanghai, China).

\subsection{Carotenoid Extraction with MTBE and HPLC Analysis}

Carotenoids in fruit juice sacs and leaves were extracted in accordance with Liu et al. [6]. In total, $1 \mathrm{~g}$ of lyophilized sample was homogenized with $15 \mathrm{~mL}$ of extraction solution (hexane/acetone/ethanol = 2:1:1, $v / v / v$, containing $0.1 \mathrm{~g} / \mathrm{L}$ butylated hydroxytoluene). After $12 \mathrm{~h}$ of saponification in darkness with $2 \mathrm{~mL}$ of $\mathrm{KOH} /$ water/methanol $(10: 25: 75, w / v / v)$, the sample was rinsed with saturated $\mathrm{NaCl}$ solution until it became neutral. After evaporation under a vacuum, the residue was redissolved in $0.6 \mathrm{~mL}$ MTBE. Carotenoid extracts were separated by HPLC (Waters 1525, Waters Co., Milford, MA, USA) equipped with a YMC C30 carotenoid column $(150 \mathrm{~mm} \times 4.6 \mathrm{~mm}, 3 \mu \mathrm{m}$; YMC, Wilmington, NC, USA) and a 2996 photodiode array detector. Methanol:acetonitrile $(1: 3, v / v)$ and MTBE were employed as eluent A and B, respectively.

The following linear gradient program was used: 0-10 min, 95:5 A:B; 10-19 min, 86:14 A:B; 19-29 min, 75:25 A:B; 29-54 min, 50:50 A:B; 54-66 min, 26:74 A:B; 67 min, 95:5 A:B.

\subsection{Limonoid Aglycone Extraction and HPLC Analysis}

In accordance with Li et al. [35] and Liu et al. [7], without any modification, bitter compounds were extracted. Briefly, with the assistance of a FexIKA vario control (IKA-Werke GmbH and Co. KG, Staufen, German), lyophilized powder of $3 \mathrm{~g}$ of juice sacs or flavedo was extracted with $50 \mathrm{~mL}$ of dichloromethane. The extraction solution was collected and dried under vacuum in an Eppendorf 5301 concentrator (Eppendorf, Hamburg, Germany) after 15 cycles of Soxhlet extraction. Finally, the residue was resolved in $1 \mathrm{~mL}$ of acetonitrile. The HPLC equipment was the same as for the carotenoid analysis, except that a C18 HPLC column $(150 \mathrm{~mm} \times 4.6 \mathrm{~mm}, 5 \mu \mathrm{m}$; Agilent, Wilmington, DE, USA) was used. A $20 \mu \mathrm{L}$ sample was injected into the HPLC and eluted with acetonitrile/100 $\mathrm{mL} \mathrm{L}^{-1}$ methanol

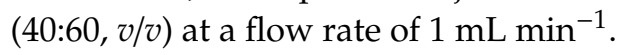

\subsection{Plant Hormone Extraction and Analysis with LC-MS}

Plant hormones were extracted in accordance with Pan et al. [36] and Ma et al. [37]. A $10 \mathrm{ng}$ aliquot of $\left[{ }^{2} \mathrm{H}_{5}\right]$ IAA, $\left[{ }^{2} \mathrm{H}_{4}\right] \mathrm{SA},\left[{ }^{2} \mathrm{H}_{2}\right] J \mathrm{~A}$, and $\left[{ }^{2} \mathrm{H}_{6}\right]$ ABA were used as internal standards. Hormones were separated by HPLC (Agilent 1100, Agilent Technologies, Palo Alto, CA, USA) and measured using an HPLC-electrometer (Applied Biosystems, Foster City, CA, USA). The MS/MS conditions for each analyte was set in accordance with Pan et al. [36].

\subsection{Volatile Compound Extraction and GC-MS Analysis}

In citrus fruit, volatile compounds are mainly accumulated in the oil glands of the peel [38]. Thus, citrus peel was used for volatile compound extractions. In accordance with Liu et al. [21], $3 \mathrm{~g}$ of peel powder was extracted in $15 \mathrm{~mL}$ MTBE containing $400 \mu \mathrm{g}$ of methyl nonanoate as an internal standard. The extraction was carried out in an ultrasonic cleaner FS60 (Fisher Scientific, Pittsburgh, PA, USA) for $1 \mathrm{~h}$ and the supernatant was collected and concentrated to $1.4 \mathrm{~mL}$ under a gentle $\mathrm{N}_{2}$ stream.

An aliquot of $1 \mu \mathrm{L}$ was analyzed by TRACE GC Ultra GC coupled with an ISQ mass spectrometer (Thermo Fisher Scientific, Waltham, MA, USA) and equipped with a TRACE ${ }^{\mathrm{TM}}$ TR-5 MS column $(30 \mathrm{~m} \times 0.25 \mathrm{~mm} \times 0.25 \mu \mathrm{m}$, Thermo Scientific, Bellefonte, PA, USA). The temperatures of the injection port, ion source, and MS transfer line were maintained at 250,260 , and $280^{\circ} \mathrm{C}$, respectively. Helium was used as a carrier gas with a split ratio of $50: 1$ at $1 \mathrm{~mL} / \mathrm{min}$. The column temperature program was initiated with $40^{\circ} \mathrm{C}$ for $3 \mathrm{~min}$, followed by a ramp of $3{ }^{\circ} \mathrm{C} / \mathrm{min}$ until $160^{\circ} \mathrm{C}$ for $1 \mathrm{~min}$, then at a rate of $5{ }^{\circ} \mathrm{C} / \mathrm{min}$ until $200^{\circ} \mathrm{C}$ for $1 \mathrm{~min}$, and finally, the temperature was raised to $240{ }^{\circ} \mathrm{C}$ at a rate of $8{ }^{\circ} \mathrm{C} / \mathrm{min}$ and maintained for $3 \mathrm{~min}$. The $\mathrm{m} / \mathrm{z}$ range of the MS scan was from 45 to 400 in positive electron ionization mode at an ionization energy of $70 \mathrm{eV}$. 


\subsection{Data Analysis}

The identification and quantification of carotenoids, bitter compounds, and volatiles were performed in accordance with Liu et al. [7,39], while the identification and quantification of plant hormones were performed in accordance with Pan et al. [36].

Significant differences in compounds between each color mutant and its corresponding control were analyzed using Student's t-test $(p<0.05)$ with SAS software (SAS Institute, Cary, NC, USA).

After autoscaling pretreatment with values of carotenoids and volatiles was done as described by van den Berg et al. [40], the package of Ggbiplot in R Version 3.5.1 software (http://www.r-project.org, R Development Core Team, Vienna, Austria) was applied to the PCA of carotenoids and volatile compounds. The package of Corrplot in R was used for the correlation analysis based on Pearson's correlation method.

Supplementary Materials: The following are available online, Figure S1: Classes and proportions of volatile compounds in fruit flavedo of Red Anliu orange (A), Anliu orange (B), Red-flesh Guanxi pummelo (C), and Guanxi pummelo (D). M: monoterpenes; MA: monoterpene alcohols; MD: monoterpene aldehydes; ME: monoterpene esters; MO: monoterpene oxides; S: sesquiterpenes; SL: sesquiterpene alcohols; SD: sesquiterpene aldehydes; SK: sesquiterpene ketone; SO: sesquiterpene oxide; AL: alcohols; AD: aldehydes. Figure S2: Heatmap dendrogram analysis based on 32 common compounds detected in fruit of four citrus varieties. S15: $\alpha$-panasinsen; S11: elixene; BC: $\beta$-carotene; TC: total carotenoids; ME2: geranyl acetate; SL3: farnesol; S8: germacrene D; M6: $\beta$-myrcene; SA: salicylic acid; NOM: nomilin; LIM: limonin; M5: $\beta$-pinene; MA8: geraniol; ABA: abscisic acid; S5: caryophyllene; 9V: 9-Z-violaxanthin; S10: valencene; M4: sabinene; AC: $\alpha$-carotene; MO4: (E)-limonene oxide; A: antheraxanthin; MA2: $\beta$-linalool; JA: jasmonic acid; M17: terpinolene; M14: (E)- $\beta$-ocimene; S4: $\beta$-elemene; M11: d-limonene; M13: (Z)- $\beta$-ocimene; MD3: geranial; MD2: neral; MA5: $\alpha$-terpineol; M2: $\alpha$-pinene. Figure S3: Principal component analysis (PCA) loading plot. A, PCA loading plot of carotenoids; B, PCA loading plot of volatile compounds. R-An, Red Anliu orange; An, Anliu orange; R-GX, Red-flesh Guanxi pummelo; GX, Guanxi pummelo. Table S1: Authentic volatile standards used and their equations of standard curves in GC-MS analysis. Table S2: Ratios of volatiles in the flavedo between red-flesh citrus mutants and their corresponding wild types.

Author Contributions: Conceptualization, J.X.; formal analysis, C.L.; investigation, C.L., M.H. and Z.W.; writing-original draft preparation, C.L.; writing—review and editing, J.X.; funding acquisition, J.X.

Funding: This research was funded by the National Key Research and Development Program, grant number 2018YFD1000200, the National Natural Science Foundation of China, grant number 31672102, and the China Postdoctoral Science Foundation, grant numbers 2015M580881 and 2016 T90952.

Acknowledgments: We thank Lesley Benyon, from Liwen Bianji, Edanz Group China (www.liwenbianji.cn/ac), for editing the English text of a draft of this manuscript.

Conflicts of Interest: The authors declare no conflict of interest.

\section{References}

1. Butelli, E.; Garcia-Lor, A.; Licciardello, C.; Las Casas, G.; Hill, L.; Recupero, G.R.; Keremane, M.L.; Ramadugu, C.; Krueger, R.; Xu, Q.; et al. Changes in anthocyanin production during domestication of Citrus. Plant Physiol. 2017, 173, 2225-2242. [CrossRef] [PubMed]

2. Hodgson, R. History. world distribution, botany and varieties. In The Citrus Industry; Reuther, W., Webber, H., Batchelor, L., Eds.; University of California Press: Berkeley, CA, USA, 1967; pp. 431-591.

3. Goodwin, T.W. Metabolism, nutrition, and function of carotenoids. Annu. Rev. Nutr. 1986, 6, $273-297$. [CrossRef] [PubMed]

4. Monselise, S.; Halevy, A. Detection of lycopene in pink orange fruit. Science 1961, 133, 1478. [CrossRef] [PubMed]

5. Saunt, J. Citrus Varieties of the World; Sinclair International Ltd.: Norwich, England, 2000; pp. 16-17.

6. Liu, Q.; Xu, J.; Liu, Y.; Zhao, X.; Deng, X.; Guo, L.; Guo, J. A novel bud mutation that confers abnormal patterns of lycopene accumulation in sweet orange fruit (Citrus sinensis L. Osbeck). J. Exp. Bot. 2007, 58, 4161-4171. [CrossRef] [PubMed]

7. Liu, C.; Yan, F.; Gao, H.; He, M.; Wang, Z.; Cheng, Y.; Deng, X.; Xu, J. Features of citrus terpenoid production as revealed by carotenoid, limonoid and aroma profiles of two pummelos (Citrus maxima) with different flesh color. J. Sci. Food Agric. 2015, 95, 111-119. [CrossRef] [PubMed] 
8. Liu, W.; Ye, Q.; Jin, X.; Han, F.; Huang, X.; Cai, S.; Yang, L. A spontaneous bud mutant that causes lycopene and $\beta$-carotene accumulation in the juice sacs of the parental Guanxi pummelo fruits (Citrus grandis (L.) Osbeck). Sci. Hortic. 2016, 198, 379-384. [CrossRef]

9. Chen, J.; Zhang, H.; Pang, Y.; Cheng, Y.; Deng, X.; Xu, J. Comparative study of flavonoid production in lycopene-accumulated and blonde-flesh sweet oranges (Citrus sinensis) during fruit development. Food Chem. 2015, 184, 238-246. [CrossRef]

10. Langenheim, J.H. Higher plant terpenoids: A phytocentric overview of their ecological roles. J. Chem. Ecol. 1994, 20, 1223-1280. [CrossRef]

11. Cutler, A.J.; Krochko, J.E. Formation and breakdown of ABA. Trends Plant Sci. 1999, 4, 472-478. [CrossRef]

12. Matusova, R.; Rani, K.; Verstappen, F.W.A.; Franssen, M.C.R.; Beale, M.H.; Bouwmeester, H.J. The strigolactone germination stimulants of the plant-parasitic Striga and Orobanche spp. are derived from the carotenoid pathway. Plant Physiol. 2005, 139, 920-934. [CrossRef]

13. Burg, S.P.; Burg, E.A. Role of ethylene in fruit ripening. Plant Physiol. 1962, 37, 179-189. [CrossRef] [PubMed]

14. Setha, S. Roles of abscisic acid in fruit ripening. Walailak J. Sci. Tech. 2012, 9, 297-308.

15. Trainotti, L.; Tadiello, A.; Casadoro, G. The involvement of auxin in the ripening of climacteric fruits comes of age: The hormone plays a role of its own and has an intense interplay with ethylene in ripening peaches. J. Exp. Bot. 2007, 58, 3299-3308. [CrossRef] [PubMed]

16. McAtee, P.; Karim, S.; Schaffer, R.; David, K. A dynamic interplay between phytohormones is required for fruit development, maturation, and ripening. Front. Plant Sci. 2013, 4, 79. [CrossRef] [PubMed]

17. Zhang, Y.; Chen, K.; Zhang, S.; Ferguson, I. The role of salicylic acid in postharvest ripening of kiwifruit. Postharvest Biol. Tec. 2003, 28, 67-74. [CrossRef]

18. Valero, D.; Díaz-Mula, H.M.; Zapata, P.J.; Castillo, S.; Guillén, F.; Martínez-Romero, D.; Serrano, M. Postharvest treatments with salicylic acid, acetylsalicylic acid or oxalic acid delayed ripening and enhanced bioactive compounds and antioxidant capacity in sweet cherry. J. Agric. Food Chem. 2011, 59, 5483-5489. [CrossRef] [PubMed]

19. Jia, H.; Zhang, C.; Pervaiz, T.; Zhao, P.; Liu, Z.; Wang, B.; Wang, C.; Zhang, L.; Fang, J.; Qian, J. Jasmonic acid involves in grape fruit ripening and resistant against Botrytis cinerea. Funct. Integr. Genom. 2016, 16, 79-94. [CrossRef] [PubMed]

20. Li, T.; Xu, Y.; Zhang, L.; Ji, Y.; Tan, D.; Yuan, H.; Wang, A. The Jasmonate-activated transcription factor MdMYC2 regulates ETHYLENE RESPONSE FACTOR and ethylene biosynthetic genes to promote ethylene biosynthesis during apple fruit ripening. Plant Cell 2017, 29, 1316-1334. [CrossRef] [PubMed]

21. Liu, C.; Cheng, Y.; Zhang, H.; Deng, X.; Chen, F.; Xu, J. Volatile constituents of wild citrus Mangshanyegan (Citrus nobilis Lauriro) peel oil. J. Agric. Food Chem. 2012, 60, 2617-2628. [CrossRef] [PubMed]

22. Li, W.; Liu, C.; He, M.; Li, J.; Cai, Y.; Ma, Y.; Xu, J. Largely different contents of terpenoids in beef red-flesh tangerine and its wild type. BMC Plant Biol. 2017, 17, 36. [CrossRef] [PubMed]

23. Niedenfuhr, S.; ten Pierick, A.; van Dam, P.T.; Suarez-Mendez, C.A.; Noh, K.; Wahl, S.A. Natural isotope correction of MS/MS measurements for metabolomics and ${ }^{13} \mathrm{C}$ fluxomics. Biotechnol. Bioeng. 2016, 113, 1137-1147. [CrossRef] [PubMed]

24. Wada, K.; Toya, Y.; Banno, S.; Yoshikawa, K.; Matsuda, F.; Shimizu, H. ${ }^{13}$ C-metabolic flux analysis for mevalonate-producing strain of Escherichia coli. J. Biosci. Bioeng. 2017, 123, 177-182. [CrossRef] [PubMed]

25. Rodrigo, M.J. Characterization of Pinalate, a novel Citrus sinensis mutant with a fruit-specific alteration that results in yellow pigmentation and decreased ABA content. J. Exp. Bot. 2003, 54, 727-738. [CrossRef] [PubMed]

26. Rahimi, S.; Devi, B.S.R.; Khorolragchaa, A.; Kim, Y.J.; Kim, J.H.; Jung, S.K.; Yang, D.C. Effect of salicylic acid and yeast extract on the accumulation of jasmonic acid and sesquiterpenoids in Panax ginseng adventitious roots. Russ. J. Plant Physl. 2014, 61, 811-817. [CrossRef]

27. Huang, J.; Cardoza, Y.J.; Schmelz, E.A.; Raina, R.; Engelberth, J.; Tumlinson, J.H. Differential volatile emissions and salicylic acid levels from tobacco plants in response to different strains of Pseudomonas syringae. Planta 2003, 217, 767-775. [CrossRef] [PubMed]

28. Hampel, D.; Mosandl, A.; Wust, M. Induction of de novo volatile terpene biosynthesis via cytosolic and plastidial pathways by methyl jasmonate in foliage of Vitis vinifera L. J. Agric. Food Chem. 2005, 53, 2652-2657. [CrossRef] [PubMed] 
29. Kim, H.J.; Chen, F.; Wang, X.; Rajapakse, N.C. Effect of methyl jasmonate on secondary metabolites of sweet basil (Ocimum basilicum L.). J. Agric. Food Chem. 2006, 54, 2327-2332. [CrossRef] [PubMed]

30. Van Schie, C.C.; Haring, M.A.; Schuurink, R.C. Tomato linalool synthase is induced in trichomes by jasmonic acid. Plant Mol. Biol. 2007, 64, 251-263. [CrossRef]

31. Talebi, M.; Moghaddam, M.; Pirbalouti, A.G. Methyl jasmonate effects on volatile oil compounds and antioxidant activity of leaf extract of two basil cultivars under salinity stress. Acta Physiol. Plant. 2018, 40, 34. [CrossRef]

32. Zhang, X.N.; Liu, J.; Liu, Y.; Wang, Y.; Abozeid, A.; Yu, Z.G.; Tang, X. Metabolomics analysis reveals that ethylene and methyl jasmonate regulate different branch pathways to promote the accumulation of terpenoid indole alkaloids in Catharanthus roseus. J. Nat. Prod. 2018, 81, 335-342. [CrossRef]

33. Patil, B.S.; Brodbelt, J.S.; Miller, E.G.; Turner, N.D. Potential health benefits of citrus: An overview. In Potential Health Benefits of Citrus; Patil, B.S., Turner, N.D., Miller, E.G., Brodbelt, J.S., Eds.; Oxford University Press: Washington, DC, USA, 2006; pp. 1-16.

34. Wang, F.; Lin, J.; Xu, L.; Peng, Q.; Huang, H.; Tong, L.; Lu, Q.; Wang, C.; Yang, L. On higher nutritional and medical properties of a carotenoid-rich mutant pomelo (Citrus maxima (L.) Osbeck). Ind. Crop. Prod. 2019, 127, 142-147. [CrossRef]

35. Li, S.J.; Wang, Z.; Ding, F.; Sun, D.; Ma, Z.C.; Cheng, Y.J.; Xu, J. Content changes of bitter compounds in 'Guoqing No.1' Satsuma mandarin (Citrus unshiu Marc.) during fruit development of consecutive 3 seasons. Food Chem. 2014, 145, 963-969. [CrossRef] [PubMed]

36. Pan, X.; Welti, R.; Wang, X. Quantitative analysis of major plant hormones in crude plant extracts by high-performance liquid chromatography-mass spectrometry. Nat. Protoc. 2010, 5, 986-992. [CrossRef] [PubMed]

37. Ma, Q.; Ding, Y.; Chang, J.; Sun, X.; Zhang, L.; Wei, Q.; Cheng, Y.; Chen, L.; Xu, J.; Deng, X. Comprehensive insights on how 2,4-dichlorophenoxyacetic acid retards senescence in post-harvest citrus fruits using transcriptomic and proteomic approaches. J. Exp. Bot. 2014, 65, 61-74. [CrossRef] [PubMed]

38. Liang, S.J.; Wu, H.; Lun, X.; Lu, D.W. Secretory cavity development and its relationship with the accumulation of essential oil in fruits of Citrus medica L. var. sarcodactylis (Noot.) Swingle. J. Integr. Plant Biol. 2006, 48, 573-583. [CrossRef]

39. Liu, C.; Jiang, D.; Cheng, Y.; Deng, X.; Chen, F.; Fang, L.; Ma, Z.; Xu, J. Chemotaxonomic Study of Citrus, Poncirus and Fortunella genotypes based on peel oil volatile compounds - deciphering the genetic origin of Mangshanyegan (Citrus nobilis Lauriro). PLoS ONE 2013, 8, e58411. [CrossRef] [PubMed]

40. Van den Berg, R.A.; Hoefsloot, H.C.; Westerhuis, J.A.; Smilde, A.K.; van der Werf, M.J. Centering, scaling, and transformations: Improving the biological information content of metabolomics data. BMC Genom. 2006, 7, 142. [CrossRef] [PubMed]

Sample Availability: Samples of the materials are available from the authors.

(C) 2019 by the authors. Licensee MDPI, Basel, Switzerland. This article is an open access article distributed under the terms and conditions of the Creative Commons Attribution (CC BY) license (http://creativecommons.org/licenses/by/4.0/). 